\title{
WHAT FRACTION OF SUN-LIKE STARS HAVE PLANETS?
}

\author{
Charles H. Lineweaver and Daniel Grether \\ Department of Astrophysics, School of Physics, University of New South Wales, Sydney, NSW 2052, Australia; \\ charley@bat.phys.unsw.edu.au \\ Received 2003 June 25; accepted 2003 August 15
}

\begin{abstract}
The radial velocities of $\sim 1800$ nearby Sun-like stars are currently being monitored by eight high-sensitivity Doppler exoplanet surveys. Approximately 90 of these stars have been found to host exoplanets massive enough to be detectable. Thus, at least $\sim 5 \%$ of target stars possess planets. If we limit our analysis to target stars that have been monitored the longest ( $\sim 15$ years), $\sim 11 \%$ possess planets. If we limit our analysis to stars monitored the longest and whose low surface activity allows the most precise velocity measurements, $\sim 25 \%$ possess planets. By identifying trends of the exoplanet mass and period distributions in a subsample of exoplanets less biased by selection effects and linearly extrapolating these trends into regions of parameter space that have not yet been completely sampled, we find that at least $\sim 9 \%$ of Sun-like stars have planets in the mass and orbital period ranges $M \sin i>0.3 M_{\text {Jup }}$ and $P<13$ years and at least $\sim 22 \%$ have planets in the larger range $M \sin i>0.1 M_{\mathrm{Jup}}$ and $P<60$ years. Even this larger area of the $\log (\operatorname{mass})-\log ($ period $)$ plane is less than $20 \%$ of the area occupied by our planetary system, suggesting that this estimate is still a lower limit to the true fraction of Sun-like stars with planets, which may be as large as $\sim 100 \%$.

Subject heading: planetary systems

On-line material: color figures
\end{abstract}

\section{INTRODUCTION}

With increasingly sensitive instruments exoplanet hunters have detected more than 100 exoplanets. The focus of these pioneering efforts has been to find and describe new exoplanets. As more exoplanets have been found, the question "What fraction of stars have planets?" has been looked at periodically. Estimates of the fraction of stars with planets can be simply calculated from the raw numbers of exoplanet hosts divided by the number of monitored stars. For example, Marcy \& Butler (2000) report that " 5\% harbor companions of 0.5 to $8 M_{\text {Jup }}$ within 3 AU." Estimates can also be based on high-precision Doppler targets in a single survey. Fischer et al. (2003) report a fraction of 15\% for high-precision Doppler targets in the original Lick sample. Estimates can also be based on a semiempirical analysis of exoplanet data (Tabachnik \& Tremaine 2002).

In this work we use a semiempirical method, staying as close to the exoplanet data as possible. We use the growth and current levels of exoplanet detection to verify that sensitive and long-duration surveys have been finding more planets in a predictable way. This is no surprise. However, quantitatively following the consistent increase of the lower limit to the fraction of stars with detected planets is an important new way to substantiate both current and future estimates of this fraction. Because of the growing importance of the question "What fraction of stars have planets?" and our increasing ability to answer this question, such a closer scrutiny of (1) the assumptions used to arrive at the answer, (2) the parameter space in which they are valid, and (3) the associated error bars is timely.

If the Sun were among the target stars of the Doppler surveys, it would be another few years before the presence of Jupiter (the most easily detected feature of our planetary system) could be confidently detected. However, we are beginning to be able to answer the question "How typical is Jupiter?" In Lineweaver \& Grether (2002) we found that extrapolations of trends found in a less biased subsample of exoplanet data indicate that Jupiter is a typical massive planet in the sense that it lies in the most densely occupied region of the $\log$ (mass)- $\log$ (period) plane. In Lineweaver, Grether, \& Hidas (2003) we updated our analysis to include exoplanets detected between 2002 January and August and found more support for this idea. However, in this previous work we made no effort to determine the absolute frequency of Jupiters or to answer the question "What fraction of stars have planets?" That is the main focus of this paper.

In $\S 2$ we present the exoplanet data set (Fig. 1). We analyze the target lists and detections and show how the fraction of stars with detected planets has increased over time (Figs. 2 and 3). In $\S 3$ we quantify how the fraction of stars with planets depends on the precision with which the radial velocity of individual stars can be measured (Fig. 4). In $\S 4$ we quantify trends in exoplanet mass and period by linear and power-law fits to histograms of a less biased subsample of exoplanets (Fig. 5). On the basis of these trends, we extrapolate into larger regions of parameter space and give estimates for the fraction of stars with planets in these regions. In $\S 5$ we summarize, discuss, and compare our results with previous work. Our approach is most similar and complementary to the work of Fischer et al. (2003) and Tabachnik \& Tremaine (2002).

\section{EXOPLANET DATA}

\subsection{Mass-Period Distribution}

Figure 1 displays the masses and periods of the 106 exoplanets detected as of 2003 June by the eight high-precision Doppler surveys analyzed in this paper. The region in the upper left labeled "Detected" is our estimate of the region in which the Doppler technique has detected virtually all the exoplanets with periods less than 3 years orbiting target stars that have been monitored for at least 3 years. This region is bounded by 3 days on the left, 3 years on the right, $13 M_{\text {Jup }}$ on the top, and at the bottom by a radial velocity of 


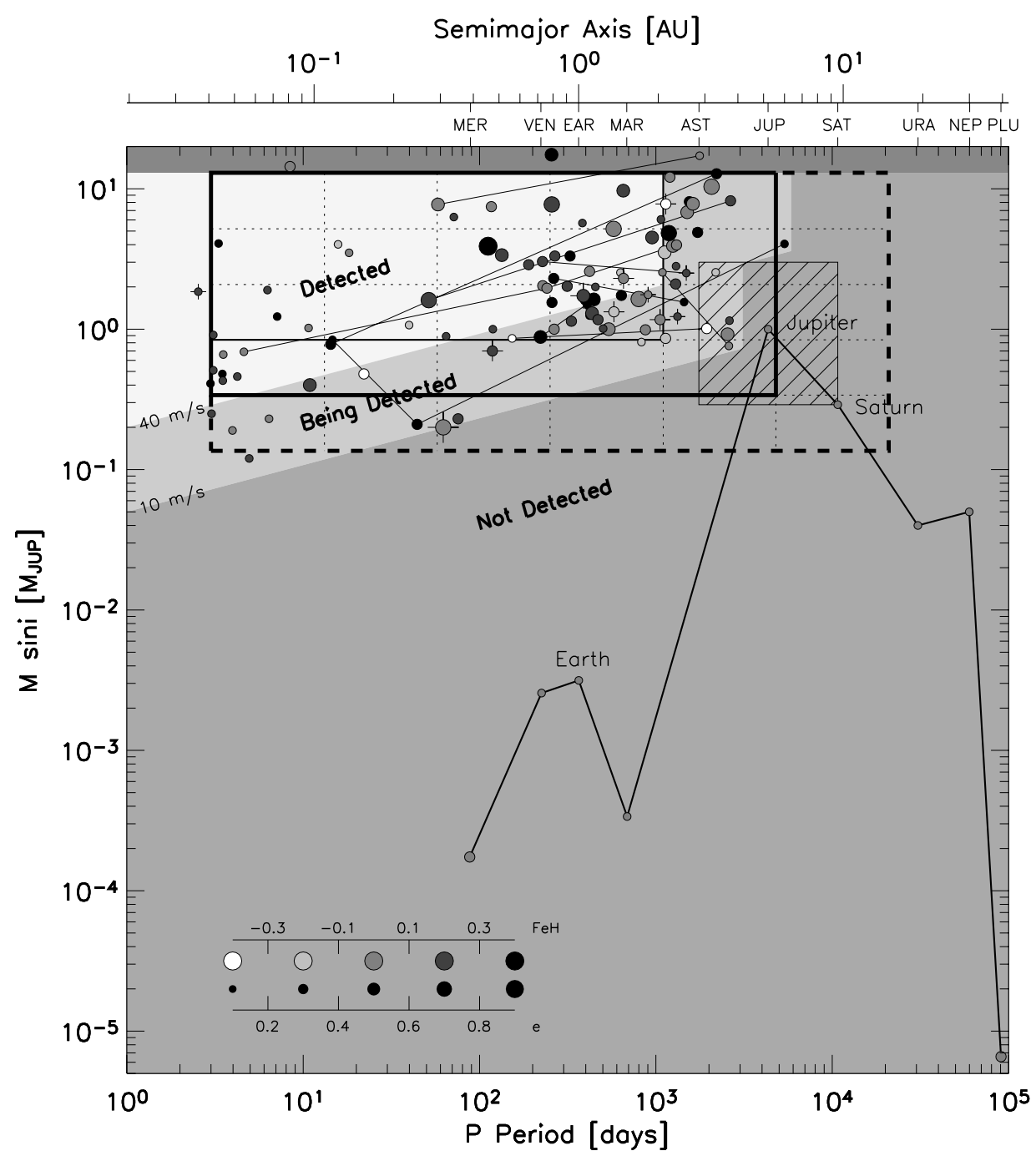

FIG. 1.-Our solar system compared to the 106 exoplanets detected by eight high-sensitivity Doppler surveys. The three regions labeled " Detected," "Being Detected," and "Not Detected "indicate the selection effects in the log(mass)-log(period) plane due to a limited time of observation and limited radial velocity sensitivity $(\S 2.1)$. The rectangular region circumscribed by the thin solid line fits almost entirely into the "Detected" region and contains what we call the less biased subsample. This subsample is the basis of the trends in mass and period identified in Fig. 5. Metallicity of the host stars is indicated by the shade of gray of the point, while eccentricity of the exoplanet orbits is indicated by point size (see key in lower left). Thin lines connect planets orbiting the same host star. The cross-hatched square is our Jupiter-like region defined by $M_{\mathrm{Sat}} \leq M \sin i \leq 3 M_{\mathrm{Jup}}$ and $P_{\text {ast }} \leq P \leq P_{\text {Sat }}$. [See the electronic edition of the Journal for a color version of this figure.].

$40 \mathrm{~m} \mathrm{~s}^{-1}$ induced in a solar-mass host star. The largest observed exoplanet period and the smallest observed radial velocity induced by a detected exoplanet are used to define the boundary between the "Being Detected" and "Not Detected" regions. The discontinuity of the "Being Detected " region near Jupiter is due to the increased sensitivity of the original Lick survey at the end of 1994. No more exoplanets should be detected in the "Detected" region unless new stars are added to the target lists. Thus, all the exoplanets marked with a "+" (detected since 2002 August) should fall in, or near, the "Being Detected" region. Of the 11 new detections since 2002 August, eight fall in the "Being Detected" region, two fall just inside the "Detected" region, while one has $P<3$ days - it was not being monitored with sufficient phase coverage for detection until recently.

We define a less biased sample of planet hosts as the 49 hosts to the planets within the rectangular region circumscribed by a thin solid line ( 3 days $<P<3$ years and $0.84<M \sin i / M_{\text {Jup }}<13$ ) in Figure 1 . This rectangle is predominantly in the "Detected" region. We will use this less biased sample as the basis for our extrapolations (§ 4). As a completeness correction for the lower right corner of this rectangle being in the "Being Detected" region, we add four planets as described in Lineweaver et al. (2003), giving us a corrected less biased sample of $53(=49+4)$ planets.

\subsection{Increasing Fraction as a Function of Monitoring Duration}

The exoplanets plotted in Figure 1 are the combined detections of eight Doppler surveys currently monitoring the radial velocities of $\approx 1812$ nearby FGK stars (Tables 3 and 4). The top panel of Figure 2 shows how the number of these target stars has increased over the past 16 years. A total of 94 stars have been found to host 106 exoplanets. Of these 94, 92 fall within our selection criteria of Sun-like stars $(=$ FGK class IV or V) with planets $\left(M \sin i<13 M_{\text {Jup }}\right)$. 


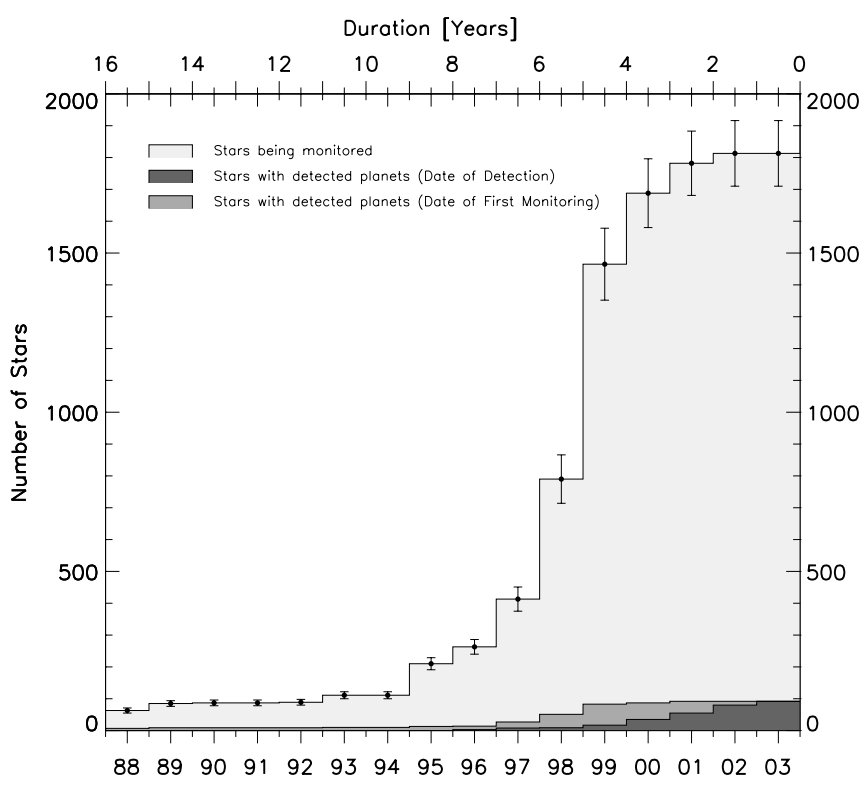

Year

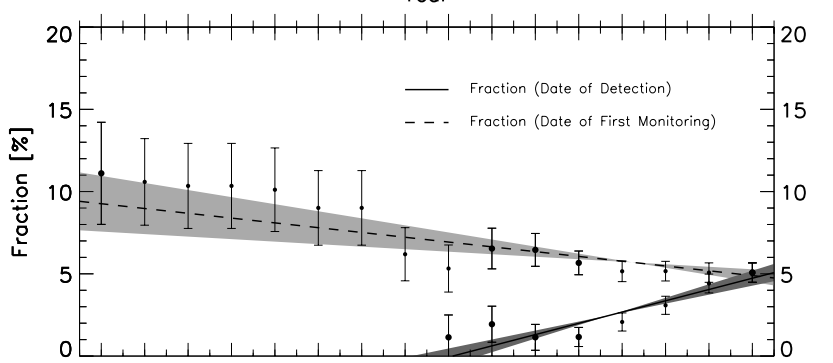

FIG. 2.-Fraction of stars with planets. Top: Histograms of the increasing number of target stars and the increasing number of them found to be hosting at least one exoplanet. The host stars are binned in two ways. In the darkest histogram they are binned at the date of detection of the first exoplanet found orbiting the star. In the middle-gray histogram they are binned at the date of first monitoring of the star. Notice the small number of target stars until the discovery of the first planet (Mayor \& Queloz 1995). Bottom: By taking the ratio, in the top panel, of the darker histograms to the lightest one, we obtain the fraction of target stars hosting at least one planet. The two binning conventions lead to different results. Using the date-of-detection binning (darkest histogram) yields the intuitive result that the fraction of host stars starts at zero in 1995 and climbs monotonically until its current value of $5 \% \pm 1 \% \quad(\approx 92 / 1812)$. The date-of-firstmonitoring binning starts on the far left at $11 \% \pm 3 \%$ from the bin of target stars that has been monitored the longest. The fraction decreases as we average in more stars that have been monitored for shorter durations. The dashed line is the result of a linear fit to a sparse, and therefore more independent, sample of the nonindependent points (data points used have a larger point size). This line yields $9 \% \pm 2 \%$ on the far left, in good agreement with the single data point in the leftmost bin. The last bin on the right is the same in both binning methods and includes all Sun-like target stars. Thus, $\approx 5 \%$ is an average fraction from target stars that have been monitored for times varying between 0 and 16 years, while $\approx 11 \%$ is the fraction from target stars that have been monitored the longest $(\approx 15$ years). The nontrivial task of estimating the total number of stars being monitored is described in Appendix A and summarized in Table 4. [See the electronic edition of the Journal for a color version of this figure.].

Six known exoplanet hosts were not included in this analysis because they were found in the context of surveys whose search strategies and sensitivities cannot easily be combined with results from the eight sensitive Doppler surveys analyzed here.

In Figure 2, the date of first monitoring and the discovery date for the detected exoplanets were largely obtained from the literature and press releases (Table 3). In some cases the date of first monitoring was estimated from the first point
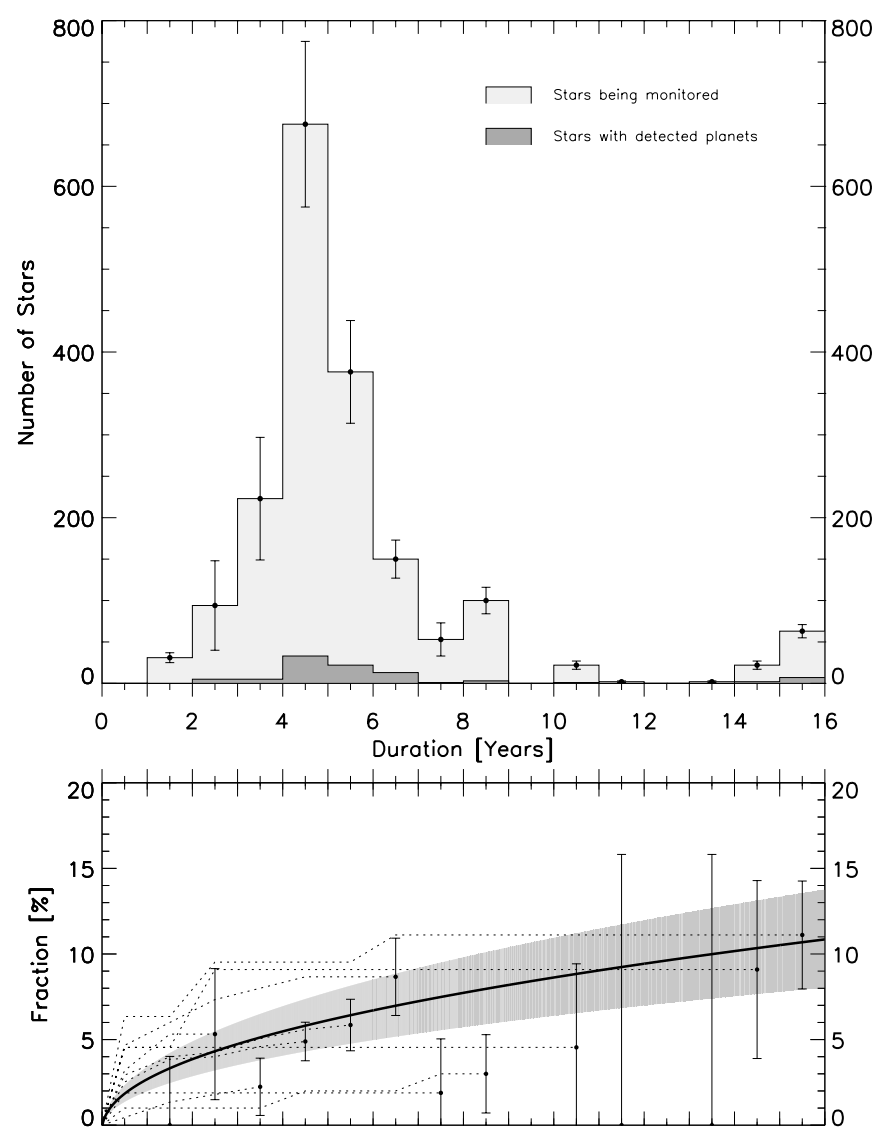

FIG. 3.-Number of targets as a function of how many years they have been monitored. Most targets have been monitored for only $\sim 5$ years. The longer a group of target stars has been monitored, the larger the fraction of planet-possessing targets. Top: Notice the small number of target stars ( 90 ) in the original Doppler surveys (bins on far right) and the $\sim 2$ years it took to start monitoring all of them. These $\sim 90(\sim 5 \%$ of the stars currently being monitored) are the only target stars that have been monitored long enough to begin to detect exoplanets with Jupiter-like masses in Jupiter-like ( $\sim 12$ year) orbital periods. This sample in the two longest duration bins has resulted in nine detections from 85 targets for a fraction of $\approx 11 \%$. It will be another 5 years before a substantially larger fraction of target stars have been monitored long enough to detect such planets. Bottom: The fractions plotted here are the ratios of the histograms in the upper panel as in the previous figure. This panel shows the effect of longer monitoring duration on the observed fraction of stars with planets. The fraction of target stars with detected planets increases with duration. This is distinct from the cumulative fractions shown in the lower panel of Fig. 2. However, the rightmost bin here is the same as the leftmost bin there. Calculation of the fraction of stars hosting exoplanets (the curve in the lower panel with the light gray $68 \%$ confidence region) is described in $\S 4.3$. The scatter of the data points around the smooth curve is due to small number statistics, varying instrumental sensitivities, and variation in observational phase coverage. For example, the two points at durations of 7.5 and 8.5 years lie below the curve, because of the lower average sensitivity $\gtrsim 10 \mathrm{~m} \mathrm{~s}^{-1}$ of the AFOE and Elodie surveys in these bins. The high point at 6.5 years is the start of the sensitive Keck survey. Thus, this plot also shows the effect of different instrumental sensitivities on the fraction of stars hosting planets. [See the electronic edition of the Journal for a color version of this figure.].

on the planet host's velocity curve. The ramp-up time needed to start observing all the stars in a survey's target list was estimated from the distribution of the date of first monitoring of a survey's detected exoplanets. That is, the rampup time needed to start observing the detected hosts was used to estimate the ramp-up time needed to start observing all the stars on a survey's target list. The exceptions to this were the time dependence of the original Lick target list obtained from Cumming, Marcy, \& Butler (1999) and the 

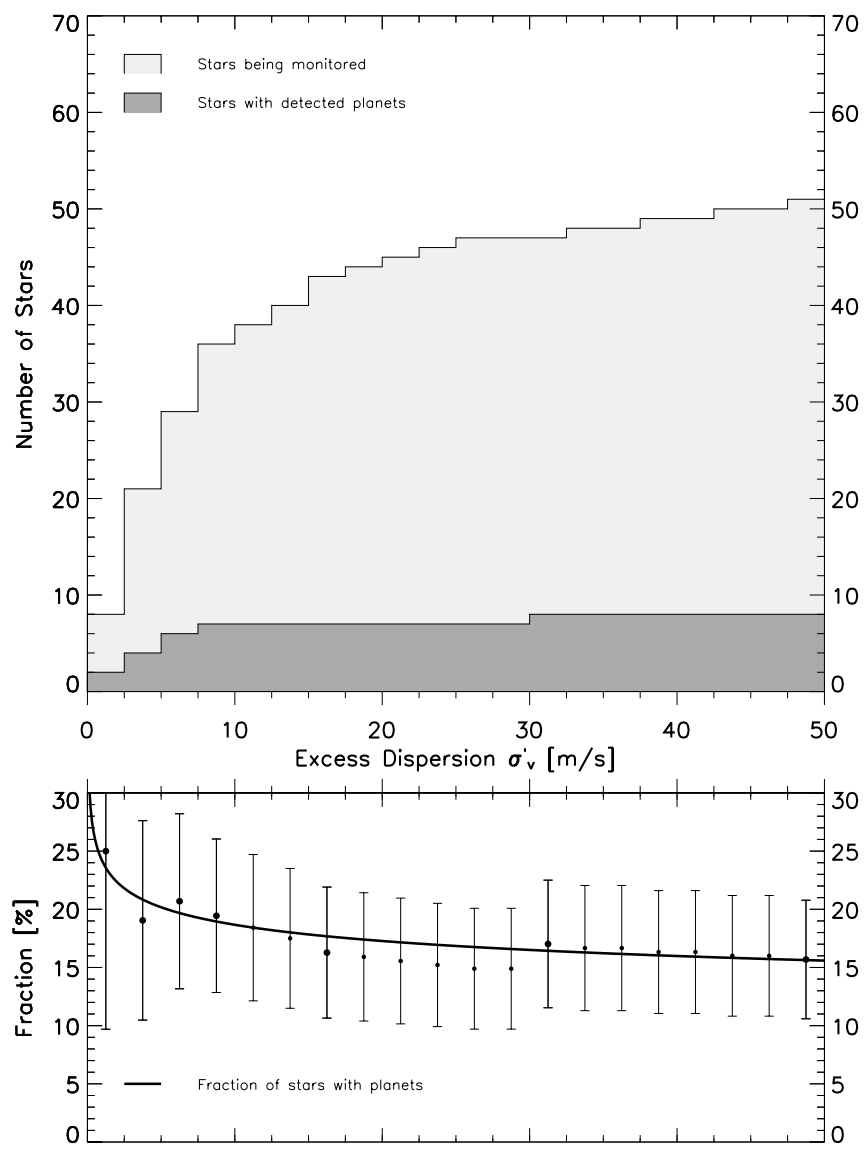

FIG. 4.-When stellar activity is low, high measurement precision is possible and a higher fraction of targets are found to host planets. Top: The number of targets in the original Lick survey that have been monitored for a duration of 14-16 years and that have an excess radial velocity dispersion $\sigma_{v}^{\prime}$ less than the value on the $x$-axis. $\sigma_{v}^{\prime}$ is a measure of how precisely one can determine the radial velocity of the targets. The number of detections from these targets is also shown. The ratio (bottom) gives the fraction of stars with planets as a function of $\sigma_{v}^{\prime}$ threshold. The $\sigma_{v}^{\prime}$ of the Lick target stars have been estimated from the stellar rotational periods tabulated by Cumming et al. (1999) using the relations $\sigma_{v}^{\prime}=10 \times\left(12 / P_{\text {rot }}\right)^{1.1}$ for $\mathrm{G}$ and $\mathrm{K}$ stars and $\sigma_{v}^{\prime}=10 \times\left(10 / P_{\text {rot }}\right)^{1.3}$ for $\mathrm{F}$ stars (Saar et al. 1998). The curve is a power-law $\left(d f / d \sigma_{v}^{\prime} \propto \sigma_{v}^{\gamma}\right)$ fit to a sparse sample of these nonindependent points (larger point size). Thus, as we select for higher Doppler precision in the Lick sample, the fraction of targets possessing planets increases from $15 \%$ to $25 \%$ for the stars with the highest precision $\left(\sigma_{v}^{\prime}<2.5 \mathrm{~m} \mathrm{~s}^{-1}\right)$. Small number statistics increases the error bars as the decreasing $\sigma_{v}^{\prime}$ threshold reduces the number of targets. In this cumulative plot the error bars are highly correlated. [See the electronic edition of the Journal for a color version of this figure.].

time dependence of the Keck target list (FGK) estimated from the FGKM histogram of Cumming et al. (2003).

The date-of-first-monitoring binning (middle-gray histogram in the top panel of Fig. 2) shows that the fraction of target stars hosting planets has a maximum of $\approx 11 \%$ from the longest monitored targets and then decreases as we average in target stars that have been monitored for shorter times. The two binning conventions used (date of detection and date of first monitoring) are equivalent today at the current value of $5 \%(\approx 92 / 1812)$.

Although this $11 \%$ estimate is based on the results from $\sim 85$ target stars of the two longest running surveys, Lick and McDonald, it is consistent with the increasing fraction based on the monitoring duration of all target stars. This is shown in Figure 3. We fit a curve to these data normalized at a duration of 15 years to the weighted average fraction of the two longest duration bins (§ 4.3). The extrapolation of this curve (based on current sensitivity) to monitoring durations of 30 years (approximate period of Saturn) yields a fraction of $\sim 15 \%$. This extrapolation corresponds to extending the white "Detected" region in Figure 1 to the upper right, above the diagonal $K=40 \mathrm{~m} \mathrm{~s}^{-1}$ line.

In the lower panel of Figure 3, we increase the fraction for a given group of stars, not when a planet is reported, but at the duration equal to the period of the newly detected planet. For example, recent detections of short-period planets increase the fraction at a duration corresponding to the short period of the newly detected planet. This smooths over artificial delays associated, for example, with not analyzing the data for the first 8 years of observations and enables us to trace with dotted lines the increasing fraction for each group of target stars.

\section{HIGH DOPPLER PRECISION TARGETS}

Instrumental sensitivity is an important limit on a Doppler survey's ability to detect planets. However, the level of stellar activity on a star's surface is also important. Using the Doppler technique, planets are more easily detected around slowly rotating stars with low-level chromospheric activity, little granulation or convective inhomogeneities, and few time-dependent surface features. To reduce these problems, some target lists have been selected for high Doppler precision (low stellar activity) by excluding targets with high values of projected rotational velocity $v \sin i$ or chromospheric emission ratio $R_{\mathrm{HK}}^{\prime}$ (Table 3).

By selecting target stars monitored the longest, the detected fraction of Sun-like stars possessing planets increased from $5 \%$ to $11 \%$. By selecting from the target stars monitored the longest those stars with the highest Doppler precision, the fraction increases still further. Fischer et al. (2003) analyzed a group of high Doppler precision target stars in the original Lick sample and found that $15 \%$ possessed planets.

We extend this idea in Figure 4 by plotting the fraction of target stars with planets as a function of their stellar activity as measured by their excess radial velocity dispersion, $\sigma_{v}^{\prime}$ (Saar, Butler, \& Marcy 1998). We find that this selection increases the fraction from $15 \%$ on the far right of Figure 4 to $\sim 25 \%$ on the far left for the highest Doppler precision stars. As long as high-precision Doppler stars are an unbiased sample of Sun-like stars, this indicates that at least $\sim 25 \%$ of Sun-like stars possess planets.

However, as we decrease the $\sigma_{v}^{\prime}$ threshold to consider only the highest precision stars, we are using fewer target stars to infer the fraction. Thus, the error bars on the estimates increase from $15 \% \pm 5 \%$ on the right to $25 \% \pm 15 \%$ on the left.

\section{FITTING FOR AND EXTRAPOLATING TRENDS}

We use extrapolation to estimate the fraction of stars with planets within regions of mass-period parameter space larger than the less biased sample (Fig. 1). Since there is no accepted theoretical model for the functional form for the mass or period distribution functions, we make simple linear fits to the histograms in $\log M \sin i$ and $\log P$, and we 

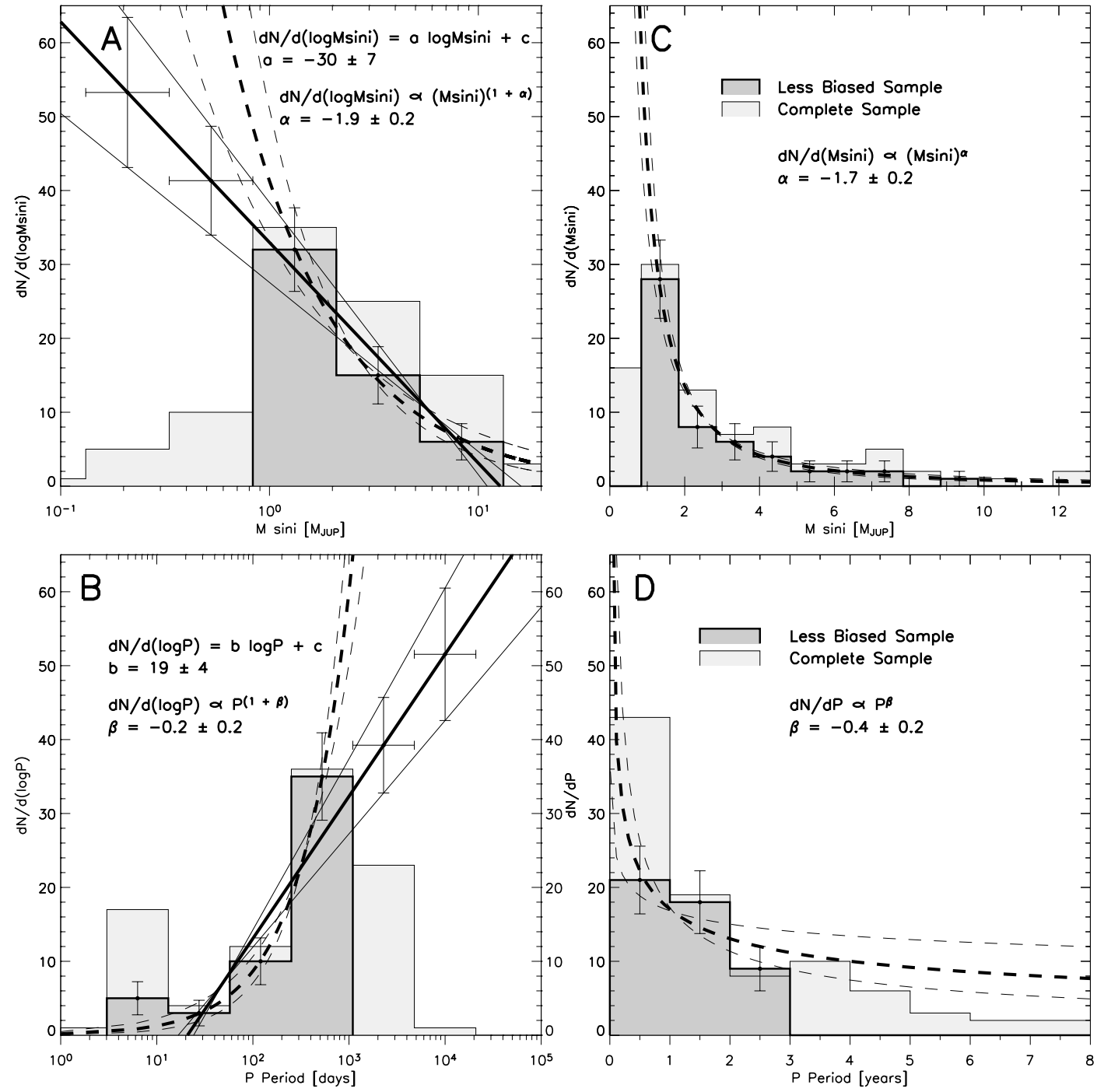

FIG. 5.-Trends that we extrapolate to lower mass and longer periods. Histograms of planet mass (top) and period (bottom) for the less biased sample of exoplanets within the thin solid rectangle of Fig. 1, compared to all exoplanet detections. Both $\log ($ left $)$ and linear (right) versions are shown. Histograms in $\log M \sin i$ and $\log P$ (panels $a$ and $b$ ) are fitted with the linear functional forms $d N / d(\log M \sin i)=a \log M \sin i+c$ and $d N / d(\log P)=b \log P+d$, where the best fits to the histograms yield $a=-30 \pm 7$ and $b=19 \pm 4$. The linearly binned histograms of $M \sin i$ and $P$ (panels $c$ and $d$ ) are fitted to the functional forms $d N / d(M \sin i) \propto(M \sin i)^{\alpha}$ and $d N / d P \propto P^{\beta}$, respectively, and we find $\alpha=-1.7 \pm 0.2$ and $\beta=-0.4 \pm 0.2$. To check the robustness of the fits, we change variables to $\log M \sin i$ and $\log P$ (effectively producing a rebinning of the data) and fit for $\alpha$ and $\beta$ in panels $a$ and $b$. We find $\alpha=-1.9 \pm 0.2$ and $\beta=-0.2 \pm 0.2$. Combining these two estimates gives our best estimates of $\alpha=-1.8 \pm 0.3$ and $\beta=-0.3_{-0.4}^{+0.3}$. In the fit of panel $b$, we ignore the five exoplanets in the smallest period bin because we are interested in the overall pattern that can be most plausibly extrapolated to larger $P$ bins, not in the pileup associated with a poorly understood stopping mechanism at $P<12$ days. For consistency, these five exoplanets have also been removed from the smallest period bin in panel $d$. [See the electronic edition of the Journal for a color version of this figure.].

fit conventional power laws to the histograms in $M \sin i$ and $P$ (Fig. 5).

Fitting

$$
d N / d(\log M \sin i)=a \log M \sin i+c
$$

to the histogram of $\log M \sin i$ (Fig. 5a), we obtain the slope $a=-30 \pm 7$. Fitting

$$
d N / d(\log P)=b \log P+d
$$

to the histogram of $\log P$ (Fig. $5 b$ ), we obtain the slope $b=19 \pm 4$. These trends are shown as thick lines. The distribution of exoplanets is not flat in either $\log M \sin i$ or $\log P$. That is, $a=-30 \pm 7$ is significantly different $(\sim 4 \sigma)$ from $a=0$ and $b=19 \pm 4$ is significantly different $(\sim 5 \sigma)$ from $b=0$. Fitting

$$
d N / d(M \sin i) \propto(M \sin i)^{\alpha}
$$

to the histogram of $M \sin i$ (Fig. 5c), we obtain $\alpha=$ $-1.7 \pm 0.2$. Fitting

$$
d N / d P \propto P^{\beta}
$$

to the histogram of $P$ (Fig. $5 d$ ), we obtain $\beta=-0.4 \pm 0.2$.

Since $d(\ln x)=d x / x$, the functional form

$$
d N / d(M \sin i) \propto(M \sin i)^{\alpha}
$$

can be written as

$$
d N / d(\log M \sin i) \propto(M \sin i)^{(1+\alpha)},
$$


TABLE 1

Best-Fit Trends in Fits to Mass and Period Histograms and Comparison with Other Analyses

\begin{tabular}{|c|c|c|c|c|}
\hline Source & $\alpha$ & $\beta$ & $a$ & $b$ \\
\hline \multicolumn{5}{|l|}{ This paper: } \\
\hline Figures $4 a$ and $4 b^{\mathrm{a}} \ldots \ldots \ldots$ & & & $-30 \pm 7$ & $19 \pm 4$ \\
\hline Figures $4 c$ and $4 d^{b}$ & $-1.7 \pm 0.2$ & $-0.4 \pm 0.2$ & $\ldots$ & $\ldots$ \\
\hline Figures $4 a$ and $4 b^{\mathrm{c}}$ & $-1.9 \pm 0.2$ & $-0.2 \pm 0.2$ & $\ldots$ & $\ldots$ \\
\hline Combined Figures $4 a-4 d \ldots$ & $-1.8 \pm 0.3$ & $-0.3_{-0.4}^{+0.3}$ & .. & $\ldots$ \\
\hline Marcy et al. 2003 & $-0.7^{\mathrm{d}}$ & $\ldots .4$ & $\ldots$ & $\ldots$ \\
\hline Lineweaver, Grether, \& Hidas 2003 .............. & $-1.6 \pm 0.2$ & $\ldots$ & $\ldots$ & $13 \pm 4$ \\
\hline 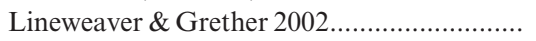 & $-1.5 \pm 0.2$ & $\ldots$ & $-24 \pm 4$ & $12 \pm 3$ \\
\hline 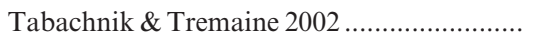 & $-1.11 \pm 0.10$ & $-0.73 \pm 0.06$ & $\ldots$ & $\ldots$ \\
\hline Stepinski \& Black 2001............ & $-1.15 \pm 0.01$ & $-0.98 \pm 0.01$ & $\ldots$ & $\ldots$ \\
\hline Jorissen, Mayor, \& Udry 2001.......................... & $\sim-1$ & $\ldots$ & $\ldots$ & $\ldots$ \\
\hline Zucker \& Mazeh 2001 & $\sim-1$ & $\ldots$ & $\ldots$ & $\ldots$ \\
\hline
\end{tabular}

a Best fit of $d N / d(\log M \sin i)=a \log M \sin i+c$ to histogram of $\log M \sin i$, and best fit of $d N / d(\log P)=b \log P+d$ to histogram of $\log P$.

${ }^{b}$ Best fit of $d N / d(M \sin i) \propto(M \sin i)^{\alpha}$ to histogram of $M \sin i$, and best fit of $d N / d P \propto P^{\beta}$ to histogram of $P$

${ }^{c}$ Best fit of $d N / d(\log M \sin i) \propto(M \sin i)^{1+\alpha}$ to histogram of $\log M \sin i$, and best fit of $d N / d(\log P) \propto P^{1+\beta}$ to histogram of $\log P$

$\mathrm{d}$ Including the lowest mass bin with no completeness corrections.

and similarly

$$
d N / d P \propto P^{\beta}
$$

can be written as

$$
d N / d(\log P) \propto P^{(1+\beta)} .
$$

We fit these functions to the histograms of $\log M \sin i$ and $\log P$ in Figures $5 a$ and $5 b$, where we find $\alpha=-1.9 \pm 0.2$ and $\beta=-0.2 \pm 0.2$. These values differ by $\sim 1 \sigma$ from the values of $\alpha$ and $\beta$ found in Figures $5 c$ and $5 d$. We attribute the differences to the different $\log$ and linear binning schemes. We combine the two values into our best estimates: $\alpha=-1.8 \pm 0.3$ and $\beta=-0.3_{-0.4}^{+0.3}$. These values are compared with other estimates in Table 1 .

If the distributions were flat in $\log M \sin i$ and $\log P$ we would find $\alpha \approx-1.0$ and $\beta \approx-1.0$, respectively. However, both the trend in mass $(\alpha)$ and in period $(\beta)$ are significantly different $(\sim 2 \sigma)$ from flat. These slopes agree with our previous results (Table 1) and show that the evidence supporting the idea that Jupiter lies in a region of parameter space densely occupied by exoplanets has gotten stronger in the sense that our new value $b=19$ is larger than our previous estimate and our new values for $a$ and $\alpha$ are more negative than our previous estimates.

The main differences between our results and previous results is that we obtain a steeper slope (more negative $\alpha$ ) when we fit the functional form $d N / d(M \sin i) \propto(M \sin i)^{\alpha}$ to the mass histogram of the data: we get $\alpha \approx-1.8$, while other analyses get $\alpha \approx-1.1$. Thus we predict more lowmass planets relative to the more easily detected number of high-mass planets than do other analyses. We obtain a more shallow slope (less negative $\beta$ ) when we fit the functional form $d N / d P \propto P^{\beta}$ to the period histogram of the data. We get $\beta \approx-0.3$, while other analyses get $\beta \sim-0.8$. Thus, we predict more hosts of long-period planets relative to the more easily detected short-period planets than do other analyses. These differences are largely due to the differences in the way the incompleteness in the lowest mass bin and the longest period bin are accounted for. This can be seen, for example, in the $\alpha=-0.7$ reported by Marcy et al. (2003) when no correction is made for incompleteness in the lowest mass bin. When we make no completeness correction and include the lowest mass bin, we reproduce their result.

\subsection{Extrapolation Using Discrete Bins}

On the basis of slopes $a$ and $b$, we make predictions for the fraction of Sun-like stars with planets within two regions of the $\log$ (mass) $-\log$ (period) plane. The estimated populations of the two lowest mass bins based on the slope $a$ are indicated in Figure $5 a$. Similarly the estimated populations of the two largest period bins based on slope $b$ are shown in Figure $5 b$. From the less biased region (Fig. 1, thin rectangle) we extrapolate in both $M \sin i$ and $P$ by one bin. When we extrapolate in $M \sin i$ or $P$, we are doing so at a fixed range in $P$ or $M \sin i$, respectively. However to estimate the fraction of stars within the thick rectangle in Figure 1 (given by the $M \sin i$ and $P$ ranges $0.3<M \sin i / M_{\text {Jup }}<13$ and 3 days $<P<13$ years) we need to estimate the number of planets $N_{M P \text { ext }}$ in the bottom right-hand region (just below Jupiter; see Fig. 1). To do this we use equation (1) (see Fig. 6). We have $N_{\mathrm{lb}}=53 \pm 2$ from Figure 1 and $N_{M \text { ext }}=41 \pm 7$ and $N_{P \text { ext }}=39 \pm 6$ from Figures $5 a$ and $5 b$. Inserting these values into

$$
\frac{N_{M \mathrm{ext}}}{N_{\mathrm{lb}}} \approx \frac{N_{M P \mathrm{ext}}}{N_{P \mathrm{ext}}}
$$

(see Fig. 6), we obtain $N_{M P \text { ext }}=30 \pm 9$. Thus, $N_{\text {hosts }}=$ $163 \pm 24$ and the fraction, $f$, of targets hosting planets is the ratio

$$
f=\frac{N_{\text {hosts }}}{N_{\text {targets }}},
$$

where $N_{\text {targets }} \sim 1812 \pm 103$ (Table 4). Finally we find $f=(163 \pm 24) / 1812 \pm 103) \approx 9 \% \pm 1 \%$. Thus, using the slopes $a$ and $b$ to extrapolate one bin into lower masses and longer periods (Fig. 1, thick solid rectangle), we find that $9 \% \pm 1 \%$ of the targeted Sun-like stars have planets. 


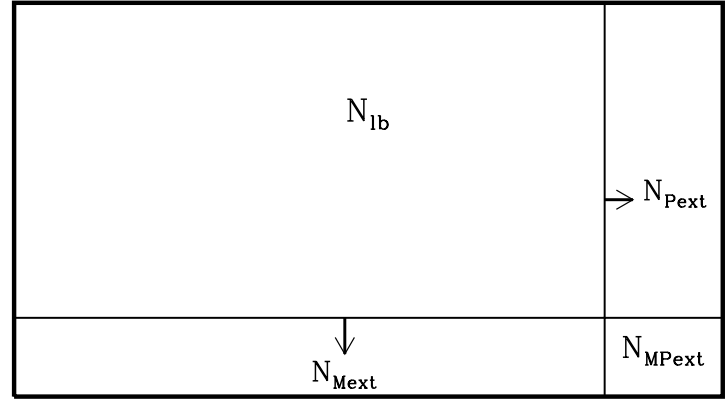

FIG. 6.- Simple method of extrapolation used to predict planet numbers in undersampled regions of the $\log ($ mass $)-\log ($ period) plane. The known value of $N_{\mathrm{lb}}$ (number of planet hosts in the less biased region of Fig. 1) and extrapolations based on the slopes $a$ and $b$ were used to derive $N_{M \text { ext }}$ and $N_{P \text { ext }}$. We then use eq. (1) to estimate $N_{M P \text { ext }}$. Our estimate of the total number of planet hosts is then $N_{\text {hosts }}=N_{\mathrm{lb}}+N_{M \text { ext }}+N_{P \text { ext }}+N_{M P \text { ext }}$.

Similarly and more speculatively we estimate the fraction contained within the thick dashed rectangle in Figure 1 by extrapolating one more bin in mass (over the same range in period as the previous mass extrapolation) and by extrapolating one more bin in period (over the same range in mass as the previous period extrapolation). The analogous numbers are $N_{\mathrm{lb}}=53 \pm 2, N_{M \text { ext }}=(41 \pm 7)+(53 \pm 10)$, and $N_{P \text { ext }}=(39 \pm 6)+(52 \pm 9)$ (where the sum of the two sets of numbers is the sum of the two separate bins). Equation (1) then yields $N_{M P \text { ext }}=161 \pm 50$. Summing the four contributions as before yields $N_{\text {hosts }}=399 \pm 84$, and thus $f=(399 \pm 84) /(1812 \pm 103) \approx 22 \% \pm 5 \%$. Thus, we estimate that $22 \% \pm 5 \%$ of the monitored Sun-like stars have planets in the larger region $\left(0.1<M \sin i / M_{\text {Jup }}<13\right.$ and 3 days $<P<60$ years) that encompasses both Jupiter and Saturn. This larger region is less than $20 \%$ of the area of the $\log$ (mass)- $\log$ (period) plane occupied by our planetary system.

\subsection{Extrapolation Using a Differential Method}

Instead of extrapolating one or two discrete bins, we can generalize to a more flexible differential method. For example, we can use the power-law functional form to integrate a differential fraction within an arbitrary range of $M \sin i$ and $P$ (Tabachnik \& Tremaine 2002):

$$
d f=c(M \sin i)^{\alpha} P^{\beta} d(M \sin i) d P .
$$

We find the normalization $c$ by inserting the known values from the less biased area:

$$
\begin{aligned}
& f=(53 \pm 2) /(1812 \pm 103)=2.9 \% \pm 0.2 \%, \\
& \alpha=-1.8 \pm 0.3, \quad \text { and } \beta=-0.3_{-0.4}^{+0.3}
\end{aligned}
$$

(Table 1). We integrate between the boundaries of the less biased rectangle (3 days $<P<3$ years and $0.84<$ $\left.M \sin i / M_{\text {Jup }}<13\right)$ and solve for the normalization. We find $c=8.6_{-5.8}^{+40.7} \times 10^{-5}$. Under the assumption that this same $c$, $\alpha$, and $\beta$ hold over larger regions of parameter space, we can integrate over the larger region and solve for $f$. For the thick solid and thick dashed regions shown in Figure 1, we find $f=20_{-10}^{+23 \%}$ and $100_{-69}^{+0} \%$, respectively. Using this differential method with the slopes $a$ and $b$, we find values nearly identical to the discrete bin extrapolations based on $a$ and $b$ : $f=9 \% \pm 1 \%$ and $22 \% \pm 5 \%$, respectively.

The power-law-based estimates are consistent with the lower linear ( $a$ - and $b$-based) estimates in the sense that the large error bars on the power-law estimates overlap with the lower estimates of the linear method. The reason that the power-law fits yield larger fractions can be seen in Figures $5 a$ and $5 b$. In the low-mass and large-period regions, the dashed curves are higher than the solid lines. Without further data from less massive planets and larger periods, we interpret this difference as an uncertainty associated with the inability of the data to prefer one of the two simple functional forms fitted here. The power-law fits are marginally better fits to the data. We interpret the lower values from the linear fits as conservative lower limits to the fraction $f$.

\subsection{Fractions in Velocity-Period Parameter Space}

The fractions discussed in $\S \S 2$ and 3 are based on exoplanet detections constrained by the Doppler technique to a trapezoidal region of the $\log$ (mass)- $\log$ (period) plane defined by a minimal velocity $K_{\min }$. The fractions discussed in the previous two sections are based on rectangular regions of the $\log$ (mass)- $\log$ (period) plane. In the lower panel of Figure 3, the fractions plotted as a function of duration come from a trapezoidal region (not a rectangular region) of the $M \sin i-P$ plane.

Hence, a fit of $d N / d P \propto P^{\beta^{\prime}}$ to the $P$ histogram of detected planet hosts will produce a value of $\beta^{\prime}$ slightly different from the $\beta$ of Figure $5 d$. We find $\beta^{\prime}=-0.5 \pm 0.2$ (while $\beta=-0.4 \pm 0.2$ ). Such a difference is expected since the number of planets at large $P$ will decrease more steeply in the trapezoidal region, because as $P$ increases, the "Detected" regions becomes narrower than a region defined by a constant $M \sin i$. The curve shown in the lower panel of Figure 3 is the integral of $P^{\beta^{\prime}}$. It is normalized to the last two bins on the right. In plotting this integral we are assuming that a survey of duration $P_{s}$ has observed a large fraction of its target stars with Doppler-detectable exoplanets of period $P \lesssim P_{s}$.

An interesting consistency check of the relationships between our extracted values of $\alpha, \beta$, and $K_{\min }$ and the limits of integration for equation (3) is described in Appendix B.

\subsection{Jupiter-like Planets}

The fraction of Sun-like stars possessing Jupiter-like planets is important since Jupiter is the dominant orbiting body in our solar system and had the most influence on how our planetary system formed.

Exoplanets with Jupiter-like periods and Jupiter-like masses are on the edge of the detectable region of parameter space. The cross-hatched square in Figure 1 is our Jupiterlike region defined by orbital periods between the period of the asteroid belt and Saturn with masses in the range $M_{\text {Sat }}<M \sin i<3 M_{\text {Jup }}$. We estimate the fraction of Sunlike stars hosting planets in this region using the differential method based on our best-fit values of $a$ and $b$. Integrating between the limits of the cross-hatched area, we find that $f=5 \% \pm 2 \%$ of Sun-like stars possess such a Jupiter-like planet. Using the differential method based on our best-fit values for $\alpha$ and $\beta$, we obtain $f=28_{-21}^{+62 \%}$. As with the different $f$ values resulting from these two methods in $\S 4.2$, these are consistent with each other and reflect the different functional forms used. 


\section{SUMMARY AND DISCUSSION}

We have analyzed the results of eight Doppler surveys to help answer the question "What fraction of stars have planets?" We use the number of targets and the number of detected planet hosts to estimate the fraction of stars with planets. Quantitatively following the consistent increase of this fraction is an important new way to substantiate both current and future estimates of this fraction. We show how the naive fraction of $\sim 5 \%$ increases to $\sim 11 \%$ when only long-duration targets are included. We extend the work of Fischer et al. (2003) by plotting the fraction as a function of excess dispersion and show how this $\sim 11 \%$ increases to $\sim 25 \%$ when only long-duration Lick targets with the lowest excess in radial velocity dispersion are considered.

We have identified trends in the exoplanet data based on a less biased subsample. We find stronger support than found previously for the idea that Jupiter-like planets are common in planetary systems (Table 1). We estimate the fraction of Sun-like stars hosting planets in a well-defined Jupiter-like region to be $\sim 5 \%$.
We have extrapolated these trends into unsampled or undersampled regions of the $\log$ (mass)- $\log$ (period) plane. We find that at least $\sim 9 \%$ of target stars will be found to host an exoplanet within the thick rectangle of Figure 1 and that more speculatively at least $\sim 22 \%$ of target stars will be found to host an exoplanet within the larger thick dashed rectangle. Our results for the fraction of Sun-like stars with planets are consistent with but are, in general, larger than previous estimates (Table 2, Fig. 7).

The largest uncertainty in this analysis is that we may be extrapolating trends derived from a small region of $\log$ (mass)- $\log$ (period) into regions of parameter space in which the trends are slightly or substantially different. This uncertainty is why we did not extrapolate beyond the dashed region in Figure 1 that contains from our solar system only Jupiter and Saturn.

It is sometimes implicitly assumed that most planetary systems will be like ours and that Earth-like planets will be common in the universe. However, as we descend in scale from galaxy, to star, to planetary system, to terrestrial planet we run more of a risk of self-selection. That is, the

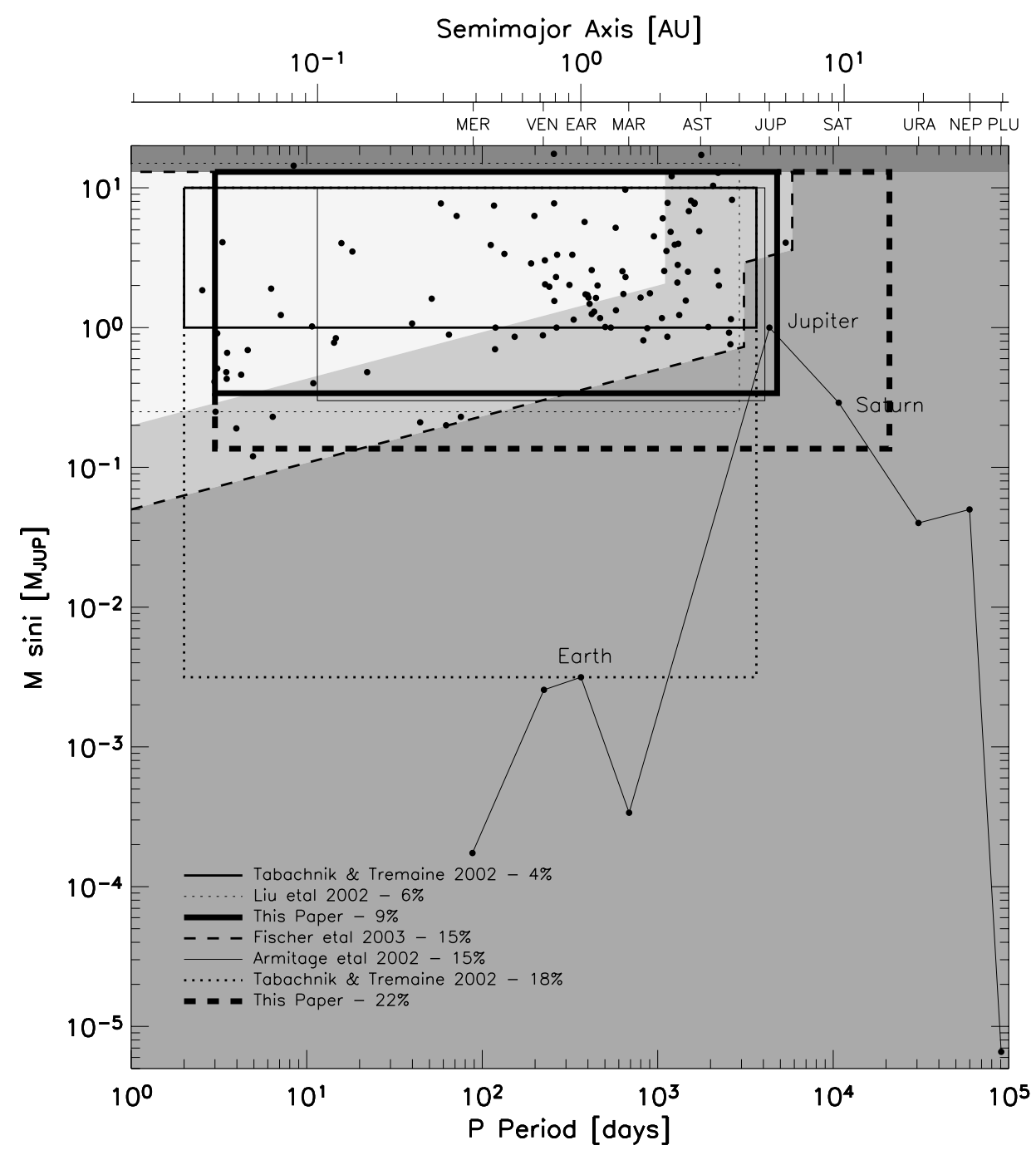

Fig. 7.-We estimate that at least $9 \%$ of Sun-like stars have planets within the thick solid rectangle and at least $22 \%$ have planets within the thick dashed rectangle $(\S 4.1)$. We find larger values by means of a differential method based on power-law fits $(\S 4.2)$. Here, we compare our results with other published estimates of the fraction of stars with planets. The papers, estimates, and the regions of the $\log M \sin i-\log P$ plane associated with these estimates are indicated. Our results are consistent with, but generally higher than, previous work (Table 2). [See the electronic edition of the Journal for a color version of this figure.]. 
TABLE 2

Fraction Comparison (See Figure 7)

\begin{tabular}{|c|c|c|c|c|c|}
\hline Source & $\begin{array}{c}M \sin i \text { Range } \\
\left(M_{\text {Jup }}\right)\end{array}$ & Period Range & $\begin{array}{c}\text { Fraction } \\
(\%)\end{array}$ & $\begin{array}{c}\text { Our Fraction }{ }^{\mathrm{a}} \\
(\%)\end{array}$ & $\begin{array}{c}\text { Our Fraction } \\
(\%)\end{array}$ \\
\hline Tabachnik \& Tremaine 2002 ............... & $1-10$ & 2 days -10 yr & 4 & $4 \pm 1$ & $6_{-2}^{+3}$ \\
\hline Liu et al. 2002 & $0.25-15$ & $0-8 \mathrm{yr}(<4 \mathrm{AU})$ & 6 & $9 \pm 3$ & $18_{-7}^{+19}$ \\
\hline Armitage et al. 2002 & $0.3-10$ & $0.03-11.2 \mathrm{yr}^{\mathrm{c}}$ & 15 & $9 \pm 3$ & $19_{-10}^{+22}$ \\
\hline Tabachnik \& Tremaine 2002 ................ & $0.003-10$ & 2 days -10 yr & 18 & $45_{-15}^{+19}$ & 100 \\
\hline
\end{tabular}

a Differential method based on slopes $a$ and $b$.

b Differential method based on powers $\alpha$ and $\beta$.

${ }^{\mathrm{c}}$ Corresponds to $0.1-5 \mathrm{AU}$.

factors that are responsible for our origin may have selected a nontypical location. Thus, answering the question "What fraction of stars have planets?" must rely on the continued analysis of the statistical distributions of exoplanets detected by the increasingly precise and ground-breaking Doppler surveys.

The hypothesis that $\sim 100 \%$ of stars have planets is consistent with both the observed exoplanet data that probe only the high-mass, close-orbiting exoplanets and with the observed frequency of circumstellar disks in both single and binary stars. The observed fractions $f$ that we have derived from current exoplanet data are lower limits that are consistent with a true fraction of stars with planets, $f_{t}$, in the range $0.25 \lesssim f_{t} \lesssim 1$. If the fraction of Sun-like stars that possess planets is representative of all stars, our result means that out of the $\sim 300$ billion stars in our Galaxy, there are between $\sim 75$ and $\sim 300$ billion planetary systems.

C. H. L. acknowledges a research fellowship from the Australian Research Council. We thank Stephane Udry, Debra Fischer, Andrew Cumming, and Sylvain Korzennik for help with many details of the exoplanet surveys. We thank Luis Tenorio for statistical counseling.

\section{APPENDIX A}

\section{ESTIMATING TARGET LIST OVERLAP}

To compute the total number of target stars being monitored (Fig. 2), we need to avoid double-counting. Target lists of six of the eight surveys considered here have been published, thus allowing the overlap in the FGK targets of these six to be eliminated by comparing target star names (Table 3). The total number monitored by these six surveys is then known $\left(N_{\text {known }} \approx 1124\right)$. The total number of targets monitored by the Coralie and Elodie surveys is known $\left(N_{C}=1100, N_{E}=350\right)$, but without published target lists the extent of overlap with the other surveys can only be estimated. We do this by using the statistics of duplicate detections. Let the total number of exoplanet hosts discovered or confirmed by Elodie and Coralie be $N_{E \text { hosts }}=16$ and $N_{\text {Chosts }}=33$, respectively. The number of these planet hosts that were discovered or confirmed by any of the other six surveys are $N_{E \text { overlap }}=8$ and $N_{\text {Coverlap }}=10$. Since there is no overlap between the Coralie and Elodie surveys (S. Udry 2003, private communication), these two sets of planet hosts are mutually exclusive. The fractional overlap of detections/confirmations is $g_{E}=N_{E \text { overlap }} / N_{E \text { hosts }}=8 / 16=0.50$ and $g_{C}=N_{\text {Coverlap }} / N_{\text {Chosts }}=10 / 33=0.30$ (Tables 3 and 4). We use these fractional detection overlaps as estimates of the fractional target list overlaps. Thus, we estimate the total number of monitored targets (excluding overlap) to be

$$
N_{\text {total }} \approx N_{\text {known }}+\left(1-g_{C}\right) N_{C}+\left(1-g_{E}\right) N_{E} .
$$

However, estimated this way, $N_{\text {total }}$ includes target stars that were found to be single-lined spectroscopic binaries (SB1). Exoplanet detection is difficult in such systems, so we correct for this by subtracting the estimated fractions of SB1s in the various surveys (between 3\% and 9\%; see Tables 3 and 4 ).

After this last step we find that $N_{\text {total }} \approx 1812 \pm 103$. The time dependence of $N_{\text {total }}$ (Fig. 2, top panel) is taken from the time dependence of $N_{\text {known }}$ and from $N_{C}$ and $N_{E}$ (Table 3) using the approximation that $g_{C}$ and $g_{E}$ are constants. Our estimates of the error associated with this procedure are indicated by the error bars on each bin in Figures 2 and 3.

\section{APPENDIX B}

\section{$M \sin i-P$ TO $K-P$ PARAMETER SPACE}

We can use the fraction $\approx 11 \%$ at a duration of 15 years in the lower panel of Figure 3 as well as the known quantities $P_{\max }$ and $K_{\min }$ to perform an interesting consistency check of the relationships between them and the extracted values of $\alpha$ and $\beta$. Assuming $M_{\text {host }} \approx M_{\odot} \gg M$ and low eccentricity, the relation between $M \sin i$ and the semiamplitude $K$ of radial velocity is

$$
K \approx A \frac{M \sin i}{P^{1 / 3}},
$$


TABLE 3

DopPler SURVEYS: TARgETS

\begin{tabular}{|c|c|c|c|c|c|c|c|c|}
\hline & AAT & Lick & Keck & Coralie & Elodie & AFOE & CES & McDonald \\
\hline Targets $^{\mathrm{a}}$. & 204 & 360 & $600^{\mathrm{b}}$ & $1100^{\mathrm{c}}$ & 350 & 146 & 37 & 33 \\
\hline FGK IV, V targets ........................ & 198 & 360 & 443 & 1100 & 350 & 136 & 32 & 33 \\
\hline List published ............................ & $\mathrm{Y}$ & $Y^{d}$ & $\mathrm{Y}^{\mathrm{d}}$ & $\mathrm{N}$ & $\mathrm{N}$ & $\mathrm{Y}^{\mathrm{e}}$ & $\mathrm{Y}$ & $\mathrm{Y}$ \\
\hline 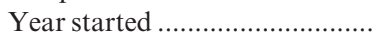 & 1998 & 1987 & 1996 & 1998 & 1994 & 1995 & 1992 & 1987 \\
\hline 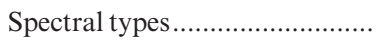 & FGKM & F7-K0 & F7-M5 & F8-M0 & FGK & FGK & F8-M5 & FGK \\
\hline 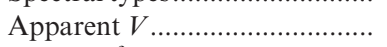 & $\lesssim 7.5$ & $\lesssim 7.5$ & $\lesssim 11$ & $<9($ for $80 \%)$ & $<7.65$ & $\lesssim 7.5$ & $\lesssim 6$ & $\lesssim 6$ \\
\hline $\log \left(R_{\mathrm{HK}}^{\prime}\right)^{\mathrm{f}} \ldots \ldots \ldots \ldots \ldots \ldots \ldots \ldots \ldots$ & $\lesssim-4.5$ & $\lesssim-4.5$ & $\lesssim-4.5$ & & & & & \\
\hline$v \sin i\left(\mathrm{~km} \mathrm{~s}^{-1}\right)^{\mathrm{g}} \ldots \ldots \ldots \ldots \ldots \ldots \ldots \ldots$ & & & & $<4^{\mathrm{c}}$ & $<5$ & $<8$ & & \\
\hline Detected SB1s $(\%)^{\mathrm{h}}$. & 8.8 & 3.3 & 3.3 & 8.7 & 3.5 & 3.5 & 8.1 & 3.5 \\
\hline 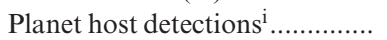 & 18 & 18 & 26 & 33 & 16 & 6 & 2 & 3 \\
\hline 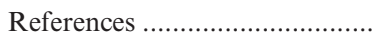 & 1 & $2,9,10$ & $3,9,11$ & 4 & 5,12 & 6 & 7 & 8 \\
\hline
\end{tabular}

Note.-AAT: Anglo Australian Observatory, Anglo Australian Telescope, UCLES Spectrograph; Lick: Lick Observatory, Hamilton Spectrograph; Keck: Keck Observatory, HIRES Spectrograph; Coralie: European Southern Observatory, Euler Swiss Telescope, Coralie Spectrograph; Elodie: Haute Provence Observatory, Elodie Spectrograph; AFOE: Whipple Observatory, AFOE Spectrograph; CES: European Southern Observatory, CAT Telescope, CES Spectrograph; McDonald: McDonald Observatory, coudé spectrograph.

${ }^{\text {a }}$ Refers to all target stars in a survey.

b Another 450 stars have one or more observations but have been dropped for various reasons (Butler et al. 2003).

c There are 550 faster rotators in a lower priority target list (S. Udry 2003, private communication).

${ }^{\mathrm{d}}$ Combined Lick/Keck (889 stars)

e S. Korzennik 2003, private communication.

${ }^{\mathrm{f}}$ The fractional Ca II H and K flux corrected for the photospheric flux (see Noyes 1984 and Saar et al. 1998).

g Projected rotational velocity.

h SB1: single-lined spectroscopic binaries. The AAT survey (Jones et al. 2002) finds that 18 out of 204 target stars are SB1. Nidever et al. 2002 mentions that 29 out of 889 Lick and Keck target stars are SB1. Endl et al. 2002 finds three out of 37 . The original Lick survey has five out of 74 (Cumming et al. 1999). We estimate the fraction of SB1s for the other surveys by noting a survey as either northern or southern hemisphere and taking an average of the known surveys in that hemisphere to estimate the fraction of SB1s. SB2s have been eliminated from the target lists. All surveys exclude binary stars when the angular separation is less than $2^{\prime \prime}$.

${ }^{\mathrm{i}}$ Including confirmations but excluding confirmations of hosts that were known to have a planet prior to the start of observation. Thus, several hosts of Lick and Elodie that are monitored by Keck and Coralie to increase phase coverage are included only in the Lick and Elodie numbers.

RefEREnCES.- (1) Jones et al. 2002. (2) Fischer et al. 2003. (3) Butler et al. 2003. (4) Udry et al. 2000. The six newest hosts discovered by Coralie and included here were announced during the XIXth IAP Colloquium on Extrasolar Planets: Today and Tomorrow (Paris, 2003 June) and papers are in preparation: Mayor et al. 2003a, Zucker et al. 2003, in preparation, and Udry et al. 2003, in preparation. (5) Sivan et al. 2000. (6) S. Korzennik 2003, private communication. (7) Endl et al. 2002. (8) Cochran \& Hatzes 1993. (9) Nidever et al. 2002. (10) Fischer et al. 1999. (11) Vogt et al. 2000. (12) M. Mayor et al. 2003, The Geneva Extrasolar Planet Search Programmes: Our Detections (http://obswww.unige.ch/ naef/planet/geneva_planets.html).

TABLE 4

Doppler Surveys: Cumulative Numbers of FGK IV-V Targets as a Function of Time

\begin{tabular}{|c|c|c|c|c|c|c|c|c|c|c|c|c|c|c|c|c|}
\hline Survey $\left(\sigma \mathrm{m} \mathrm{s}^{-1}\right)^{\mathrm{a}}$ & $1988^{\mathrm{b}}$ & 1989 & 1990 & 1991 & 1992 & 1993 & 1994 & 1995 & 1996 & 1997 & 1998 & 1999 & 2000 & 2001 & 2002 & 2003 \\
\hline Lick $(10-15)$. & 44 & 56 & 62 & 64 & 66 & 67 & 73 & $\cdots$ & $\ldots$ & $\cdots$ & .. & & & & & \\
\hline Lick $(3-5) \ldots \ldots$ & $\ldots$ & $\ldots$ & $\ldots$ & $\ldots$ & $\ldots$ & $\ldots$ & $\ldots$ & 74 & 74 & 74 & 265 & 360 & 360 & 360 & 360 & 360 \\
\hline McDonald (10-20)....... & 22 & 33 & 33 & $\ldots$ & $\ldots$ & $\ldots$ & $\ldots$ & $\ldots$ & $\ldots$ & $\ldots$ & $\ldots$ & $\ldots$ & $\ldots$ & $\ldots$ & $\ldots$ & \\
\hline McDonald (5-10)......... & $\ldots$ & $\ldots$ & $\ldots$ & 33 & 33 & 33 & 33 & 33 & 33 & 33 & 33 & $\ldots$ & $\ldots$ & $\ldots$ & $\ldots$ & \\
\hline McDona & $\ldots$ & $\ldots$ & $\ldots$ & $\ldots$ & $\ldots$ & $\ldots$ & $\ldots$ & & & . & - & 33 & 33 & 33 & 33 & 33 \\
\hline . & 0 & 0 & 0 & 0 & 0 & 29 & 29 & 29 & 29 & 32 & 32 & 32 & 32 & & 2 & 32 \\
\hline $\mathrm{El}$ & 0 & 0 & 0 & 0 & 0 & 0 & 0 & 142 & 142 & 15 & 31 & 35 & 350 & 350 & 350 & 350 \\
\hline 0$)$... & 0 & 0 & 0 & 0 & 0 & 0 & 0 & 23 & 90 & 113 & 13 & 136 & 136 & 136 & 136 & 136 \\
\hline $\mathrm{k}$ & 0 & 0 & 0 & 0 & 0 & 0 & 0 & 0 & 8 & 179 & 270 & 326 & 360 & 399 & 443 & 443 \\
\hline $\operatorname{AAT}(\sim$ & 0 & 0 & 0 & 0 & 0 & 0 & 0 & 0 & 0 & 0 & 66 & 198 & 198 & 198 & 198 & 198 \\
\hline Coralie $(\sim 3$ & 0 & 0 & 0 & 0 & 0 & 0 & 0 & 0 & 0 & 0 & 33 & 700 & 1000 & 1100 & 1100 & 1100 \\
\hline Cumul & 66 & 89 & 95 & 97 & 99 & 129 & 135 & 301 & 376 & 590 & 1150 & 2135 & 2469 & 2608 & 2652 & 2652 \\
\hline No overlap ${ }^{c} \ldots \ldots \ldots \ldots \ldots \ldots$ & 63 & 85 & 87 & 87 & 89 & 111 & 111 & 210 & 263 & 413 & 790 & 1465 & 1688 & 1782 & 1812 & 1812 \\
\hline Noncumulative ............ & 66 & 23 & 6 & 2 & 2 & 30 & 6 & 166 & 75 & 214 & 560 & 985 & 334 & 139 & 44 & 0 \\
\hline 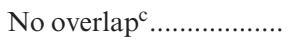 & 63 & 22 & 2 & 0 & 2 & 22 & 0 & 99 & 53 & 150 & 377 & 675 & 223 & 94 & 30 & 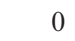 \\
\hline
\end{tabular}

a Internal error also known as instrument sensitivity.

${ }^{\mathrm{b}}$ Binning is from July to June with January at the center of bin.

c Values have been corrected for overlapping target lists and for estimated numbers of single-lined spectroscopic binaries (SB1). 
where $A=200$ when $K$ is expressed in units of $\mathrm{m} \mathrm{s}^{-1}, M \sin i$ in $M_{\mathrm{Jup}}$, and $P$ in days. We are interested only in exoplanets with $M \sin i<13 M_{\text {Jup }}$ (approximately the deuterium-burning limit). Using equation (B1) to change variables from $M \sin i$ to $K$, we can write

$$
d f=c(M \sin i)^{\alpha} P^{\beta} d(M \sin i) d P
$$

(eq. [3]) as

$$
d f=\frac{c}{A^{(1+\alpha)}} K^{\alpha} P^{\beta+(1 / 3)(1+\alpha)} d K d P .
$$

Under the assumption that $M \sin i$ is uncorrelated with $P$ and that $\alpha$ and $\beta$ are approximately constant within the region of interest, we can integrate between arbitrary limits. The upper limit on $K$ depends on period: $K_{\max }(P)=13 A / P^{1 / 3}$ from equation (B1). We then have

$$
f=\frac{c}{(1+\alpha) A^{(1+\alpha)}}\left[(13 A)^{(1+\alpha)} \int_{0}^{P_{\max }} P^{\beta} d P-K_{\min }^{(1+\alpha)} \int_{0}^{P_{\max }} P^{\beta+(1 / 3)(1+\alpha)} d P\right] .
$$

Using our best estimates $\alpha=-1.8, \beta=-0.3, c=8.6 \times 10^{-5}$, and $f=0.106$ from the lower panel in Figure 3 at 15 years, we integrate equation (B4) and solve for $K_{\min }$. We find $K_{\min }=16_{-4}^{+6} \mathrm{~m} \mathrm{~s}^{-1}$. The weighted average internal error of the original Lick and McDonald observing programs that contain the 85 stars in these two bins is $\sigma=6-10 \mathrm{~m} \mathrm{~s}^{-1}$. The minimum signal-to-noise ratio of exoplanet detections is $\sim 3$, so we expect $K_{\min } \approx 3 \sigma$, which is indeed the case.

\section{REFERENCES}

Armitage, P. J., Livio, M., Lubow, S. H., \& Pringle, J. E. 2002, MNRAS, 334,248

Butler, P. R., Marcy, G. W., Vogt, S. S., Fischer, D. A., Henry, G. W., Laughlin, G., \& Wright, J. T. 2003, ApJ, 582, 455

Cochran, W. D., \& Hatzes, A. P. 1993, in ASP Conf. Ser. 36, Planets around Pulsars, ed. J. A. Phillips, S. E. Thorsett, \& S. R. Kulkarni (San Francisco: ASP), 267

Cumming, A., Marcy, G. W., \& Butler, P. R. 1999, ApJ, 526, 890

Cumming, A., Marcy, G. W. Butler, R. P. \& Vogt, S. S. 2003, in ASP Conf. Ser. 294, Scientific Frontiers in Research on Extrasolar Planets, ed. D. Deming \& S. Seager (San Francisco: ASP), 27

Endl, M., Kürster, M., Els, M., Hatzes, A. P., Cochran, W. D., Dennerl, K., \& Doebereiner, S. 2002, A\&A, 392, 671

Fischer, D. A., Butler, P. R., Marcy, G. W., Vogt, S. S., \& Henry, G. W. 2003, ApJ, 590, 1081

Fischer, D. A., Marcy, G. W., Butler, P. R., Vogt, S. S., \& Apps, K. 1999, PASP, 111,50

Jones, H. R. A., Butler, P. R., Marcy, G. W., Tinney, C. G., Penny, A. J., McCarthy, C., \& Carter, B. D. 2002, MNRAS, submitted (astro-ph/0206216)

Jorrisen, A., Mayor, M., \& Udry, S. 2001, A\&A, 379, 992

Lineweaver, C. H., \& Grether, D. 2002, Astrobiol., 2, 3, 325

Lineweaver, C. H., Grether, D., \& Hidas, M. 2003, in ASP Conf. Ser. 294, Scientific Frontiers in Research on Extrasolar Planets, ed. D. Deming \& S. Seager (San Francisco: ASP), 161
Liu, M. C., Fischer, D. A., Graham, J. R., Lloyd, J. P., Marcy, G. W., \& Butler, P. R. 2002, ApJ, 571, 519

Marcy, G. W., \& Butler P. R. 2000, PASP, 112, 137

Marcy, G. W. Butler, P. R., Fischer, D. A., \& Vogt, S. S. 2003, in ASP Conf. Ser. 294, Scientific Frontiers in Research on Extrasolar Planets, ed. D. Deming \& S. Seager (San Francisco: ASP), 1

Mayor, M., \& Queloz, D. 1995, Nature, 378, 355

Mayor, M., Udry, S., Naef, D., Pepe, F., Queloz, D., Santos, N. C., \& Burnet, M. 2003, A\&A, submitted

Nidever, D. L., Marcy, G. W., Butler, P. R., Fischer, D. A., \& Vogt, S. S. 2002, ApJS, 141, 503

Noyes, R. W., Hartmann, L. W., Baliunas, S. L., Duncan, D. K., \& Vaughan, A. H. 1984, ApJ, 279, 763

Saar, S. H., Butler, P. R., \& Marcy, G. W. 1998, ApJ, 498, L153

Sivan, J. P., Mayor, M., Naef, D., Queloz, D., Udry, S., Perrier-Bellet, C., \& Beuzit, J. L. 2000, in IAU Symp. 202, Planetary Systems in the Universe: Observation, Formation and Evolution, ed. A Penney, P. Artymowicz, A.-M. Lagrange, \& S. Russell (San Francisco: ASP), in press

Stepinski, T. F., \& Black, D. C. 2000, A\&A, 356, 903

Tabachnik, S., \& Tremaine, S. 2002, MNRAS, 335, 151

Udry, S., Mayor, M., Naef, D., Pepe, F., Queloz, D., Santos, N. C., Burnet, M., Confino, B., \& Melo, C. 2000, A\&A, 356, 590

Vogt, S. S., Marty, G. W., Butler, P. R., \& Apps, K. 2000, ApJ, 536, 902

Zucker, S., \& Mazeh, T. 2001, ApJ, 562, 1038 